\title{
Research of processes of the heatmass transfer in the porous environments having stochastic characteristics on the basis of methods of applied synergetic
}

\author{
Ludmila Larina ${ }^{1}$, Dmitryi Ruslyakov ${ }^{1}$, Olga Tikhonova ${ }^{1,{ }^{*}}$, and Boris Kalmykov ${ }^{1}$ \\ ${ }^{1}$ Institute of Service and Entrepreneurship (branch) "Don State Technical University" in Shakhty, 147, \\ st. Shevchenko, 346500, Shakhty, Russia
}

\begin{abstract}
On the basis of a synergetic approach, mathematical models of the stochastic similarity of the functioning of heat and mass transfer processes in porous media (grain materials) have been developed. In these models, the indicators of the stochastic characteristics of these media are combined with the parameters of the processes of hygrothermal treatment under vacuum conditions: residual pressure - $\mathrm{P}$, temperature - T, time- $\tau$, with a density of couple - $\rho$. The resulting models can be used to control hygrothermal processes in the processing of natural tanning and grain materials that have a stochastic character of the building. A method for the formation of mathematical models of stochastic similarity has been developed, including functional dependences of indicators of stochastic characteristics of materials subjected to hygrothermal treatment on parameters characterizing its state: input, setting, disturbing influences and internal (structural) system.
\end{abstract}

\section{Introduction}

The synergy method based on a triad "nonlinearity-multidimensionality-multiplying" can be used for management of processes of gigrotermichesky processing in the conditions of a vacuum of the natural tanning and grain materials having the stochastic nature of the building.

\section{Methods}

The essence of the synergetic approach is that the object of study is considered as a complex self-organizing open non-linear biotechnological system consisting of a bio-object - grains such as oats and technological means, between which there are direct and feedback connections. The control of these bonds based on their analysis and synthesis leads to acceleration of the mechanisms of heat and mass transfer in porous media with stochastic

${ }^{*}$ Corresponding author: tudd@mail.ru 
characteristics, which leads to self-organization of the bioobject structure and its transfer to a new state, characterized by the selected output characteristic.

The technique of forming of mathematical models of stochastic similarity, includes functional dependences of indicators of stochastic characteristics of the materials subjected to gigrotermichesky processing from the parameters characterizing its status: the entrance, setting, perturbing influences and internal (structural) [1,2]. The developed mathematical models will allow to consider a possible deviation of parameters of processing from their rated (recommended) values, that is to consider stochastic similarity of functioning of the system consisting of the following subsystems:

- "means of technology" (camera, vacuum pump and steam generator);

- "technology" (sequence of execution gigrotermicheskikh of operations: moistening, wet and thermal fixing, drying and wet thermal treatment);

- "a technology object" (grain samples placed in the vacuum chamber);

- "products" (grain samples processed after appropriate hygrothermal exposure).

As an example it is given, the created according to the developed technique, functional dependence of an output characteristic of process of vacuum and sorption moistening from parameters of the considered system [3-5].

Thus, in order to ensure the quality of the hygrothermal treatment of grain materials, evaluated by certain indices of physical and mechanical properties and having a stochastic nature of the structure, it is necessary to take into account the possible deviation of the processing parameters - $\mathrm{P}, \mathrm{T}$.

The developed method of forming mathematical models of stochastic similarity provides for:

1) Obtaining functional dependence of the output characteristic of the system on parameters of input, setting, perturbating effects and internal (structural) parameters of the system that determine its state.

2) Generation by method of zero dimensions (as a rule) of expressions for private criteria of similarity according to the initial functional dependence.

3) Combining the resulting expressions for private similarity criteria by physical significance and target [6].

4) Determination of numerical values of combined criteria by nominal values of parameters of IGO subsystems functioning in order to form generalized criterion expressions.

5) Obtaining from generalized criterion expressions the output characteristic of the subsystem.

6) Setting of boundary values for output characteristic of IHO [7] subsystems and performance parameters.

7) Determination of value of output characteristic of a subsystem ( $(i)$ in stochastic sense by a probabilistic method, proceeding from possible deviations of independent parameters (XJ, ZJ, SJ) from their nominal values because of the stochastic structure of skin. To do this, the resulting generalized criterion expression is decomposed into a Taylor series and used to calculate linear members of that series:

$$
Y_{i}=f_{i}\left(\pi_{\mathrm{yi}}, X_{j}, Z_{j}, S_{j}\right)=\pi_{\mathrm{yi}}\left[f_{i}\left(M_{\mathrm{xj}}, M_{\mathrm{Zj}}, M_{\mathrm{sj}}\right)+\frac{\partial f_{i}}{\partial X_{j}}\left(X_{j}-M_{\mathrm{xj}}\right)+\frac{\partial f_{i}}{\partial Z_{j}}\left(Z_{j}-M_{\mathrm{zj}}\right)+\frac{\partial f_{i}}{\partial S_{j}}\left(S_{j}-M_{\mathrm{Sj}}\right)\right]
$$

where Mxj, Mzj , Msj - mathematical expectations of independent parameter values; $\frac{\partial f_{i}}{\partial X_{j}}, \frac{\partial f_{i}}{\partial Z_{j}}, \frac{\partial f_{i}}{\partial S_{j}}$ - private derivative functions of communication by independent parameters.

Nominal values of independent parameters are taken as mathematical expectations.

8) Construction of graphical dependencies of output characteristic of funk-cation on independent parameters taking into account stochastic structure of grain. 
9) Determination of the area of guaranteed hygrothermic effect on grain materials taking into account their stochastic structure [8-13].

10) Check of possibility of assignment of minimum processing modes in time from comparison of sum of relative deviations (errors) of independent parameters from their nominal values in connection with stochastic skin structure with limit of relative tolerances for change of output characteristic of the considered subsystem:

$$
\delta_{x_{j}}=\frac{X_{j}-X_{j_{0}}}{X_{j}} ; \delta_{Z_{j}}=\frac{Z_{j}-Z_{j_{0}}}{Z_{j}} ; \delta_{S_{j}}=\frac{s_{j}-S_{j_{0}}}{S_{j}},
$$

By way of example, a function of the output characteristic of the vacuum sorption humidification process, which is in the form of:

$$
\mathrm{EK}=\mathrm{f}(\mathrm{d}, \mathrm{m} 3, \mathrm{VM} . \mathrm{K}, \mathrm{Q}, \mathrm{CK}, \mu, \varphi \text { П.C, TH, TK, PH, PK, } \tau, \mathrm{QT}, \mathrm{VK}, \mathrm{D}, \rho, \mathrm{C} \Pi \mathrm{O}, \mathrm{R} \Pi \text { ) (3) }
$$

Taking into account this dependence, according to the method considered, there were obtained partial p-criteria of similarity, combined by physical significance (Table 3 ).

To obtain:

criterion - $\pi \mathrm{Ki}$ private criteria united: $\pi \mathrm{Q} \Pi, \pi \mathrm{Ck}, \pi \mu, \pi \mathrm{d}, \pi \mathrm{m} 3, \pi \mathrm{Ek}, \pi \mathrm{Ck}$;

criterion - $\pi \mathrm{Ti}$ private criteria: $\pi \mathrm{Tk}, \pi \mathrm{Pk}, \pi \mathrm{D}, \pi \mathrm{I}$;

criteria - $\pi \mathrm{Ci}$ private criteria were combined with the individual criteria $\pi \mathrm{Vk}, \pi \mathrm{C} п$, $\pi \mathrm{Q}$.

The combined criteria obtained under the accepted unambiguous conditions (Table 1) for the "humidification" subsystem and unambiguous conditions for other subsystems of the intensified hygrothermal treatment and, based on the condition of similarity of operation of the considered subsystems:, $\pi_{i}=$ idem $=$ const. are given in Table 1 .

Table 1. Criteria of similarity of functioning of subsystems of the system of intensified hygrothermal

\begin{tabular}{|c|c|c|c|c|}
\hline $\begin{array}{c}\text { Subsystems } \\
\text { Objedi } \\
\text { Known } \\
\text { Crime } \\
\text { Terium of } \\
\text { similarity } \\
\end{array}$ & "Moistening" & "Drying" & $\begin{array}{l}\text { "Damp and } \\
\text { thermal } \\
\text { processing" }\end{array}$ & "Fixing" \\
\hline 1 & 2 & 3 & 4 & 5 \\
\hline \multirow[t]{2}{*}{$\begin{array}{l}\pi_{K i}-\text { criteria } \\
\text { for efficiency } \\
\text { of processing }\end{array}$} & $\begin{array}{c}\pi_{\mathrm{Ky}}= \\
\frac{\mathrm{P}_{\mathrm{H}}^{3.5} \cdot \tau^{2}}{\mathrm{~T}_{\mathrm{H}} \cdot \rho^{0.5} \mathrm{Ek}} \mathrm{A} \\
50.7\end{array}$ & $\begin{array}{c}\pi_{\mathrm{kc}}= \\
\frac{\mathrm{P}_{\mathrm{H}}^{3} \cdot \tau^{1} \cdot \rho}{\mathrm{T}_{\mathrm{H}} \cdot \Delta k} \\
70.4\end{array}$ & $\begin{array}{c}\pi_{\mathrm{kBTo}}= \\
\frac{\mathrm{P}_{\mathrm{H}}^{2.5} \cdot \tau^{2}}{\mathrm{~T}_{\mathrm{H}} \cdot \rho^{0.5} \cdot \Delta k} \\
15.5\end{array}$ & $\begin{array}{c}\pi_{\mathrm{kf}}= \\
\frac{\mathrm{P}_{\mathrm{H}}^{3} \cdot \tau \cdot \rho 3}{\mathrm{~T}_{\mathrm{H}} \cdot \Delta k} \mathrm{~A} \\
80 \cdot 10^{-4}\end{array}$ \\
\hline & \multicolumn{4}{|c|}{$A=\frac{d}{m} \cdot \frac{1}{c_{k} \cdot Q_{n} \cdot \mu}$} \\
\hline \multirow{3}{*}{$\begin{array}{l}\pi_{T i-\text { process }} \\
\text { intensity } \\
\text { criterion }\end{array}$} & \multicolumn{3}{|c|}{$\pi_{\mathrm{Ti}}=\frac{\mathrm{T}_{K} \cdot \mathrm{P}_{\mathrm{H}}^{1.5}}{\mathrm{~T}_{\mathrm{H}} \cdot \mathrm{P}_{K} \cdot D \cdot \tau \cdot \sqrt{\rho}}$} & $\pi_{\mathrm{Tf}}=\frac{\mathrm{P}_{\mathrm{H}}^{2}}{\mathrm{~T}_{k} \cdot p^{2}}$ \\
\hline & $\pi_{T y}=5494$ & $\pi_{T_{c}}=4797$ & $\pi_{T_{s m o}}=15550$ & $\pi_{\mathrm{Tf}}=3.91$ \\
\hline & \multicolumn{4}{|c|}{$\begin{array}{l}\text { At combination of operations } \\
=\mathrm{T}_{\mathrm{H}} \quad \frac{\mathrm{P}_{K}}{=}=1.78\end{array}$} \\
\hline \multirow{2}{*}{$\begin{array}{l}\pi_{C i}-\text { criterion } \\
\text { of suitability } \\
\text { to a giving } \\
\text { obor for } \\
\text { concrete } \\
\text { operation }\end{array}$} & \multicolumn{4}{|c|}{$\pi_{\mathrm{Ci}}=\frac{V_{K} \cdot \mathrm{P}_{\mathrm{H}}^{2}}{Q_{\mathrm{T}} \cdot \mathrm{C}_{\Pi 0} \cdot \rho}$} \\
\hline & $\pi_{\mathrm{Cy}}=34.6$ & $\pi_{\mathrm{Cc}}=38.3$ & $\pi_{\text {Свто }}=36$ & $\pi_{\mathrm{Cf}}=38.3$ \\
\hline
\end{tabular}
treatment using vacuum combined by the target task. 
An expression for the final (after treatment) modulus of elasticity of the skin of the $\pi \mathrm{Ky}$ is obtained from the generalized criterion expression for the efficiency criterion grain of EK:

$$
E_{K}=\frac{1}{\pi_{\mathrm{Ky}}} \cdot \frac{d}{m_{3}} \cdot \Pi \cdot \frac{1}{C_{K} Q_{n} \mu} \cdot \frac{\mathrm{PH}^{3,5} \tau^{2}}{\mathrm{Tн} \rho^{0,5}},
$$

where P - conditional microcapillary porosity grain shell.

The boundary values of the output characteristic of the operation of the "humidification" subsystem and independent parameters are determined on the basis of experimental studies [1]:

$$
\left.\left.\left.\left.10^{6} \cdot 17,9 \leq E \mathrm{k} \leq 24,1 \cdot 10^{6} ; \mid\right\} 0,02 \cdot 10^{6} \leq P \leq 0,05 \cdot 10^{6} ; \mid\right\} 328 \leq T \leq 333 ; \mid\right\} 120 \leq \tau \leq 360, \mid\right\}
$$

After the Taylor series decomposition of equation (1), the value of the output characteristic of the subsystem in the stochastic sense is determined by substituting in the following expression the numerical values of its components:

$$
\begin{gathered}
E_{\mathrm{k} i}=E_{\mathrm{k}}+\frac{A}{\pi_{\mathrm{ky}}} \cdot \Pi \cdot\left[\frac{\partial f}{\partial P} \cdot\left(P_{\mathrm{cp}}-P_{i}\right)+\frac{\partial f}{\partial T} \cdot\left(T_{\mathrm{cp}}-T_{i}\right)+\frac{\partial f}{\partial \tau} \cdot\left(\tau_{\mathrm{cp}}-\tau_{i}\right)+\frac{\partial f}{\partial \rho} .\right. \\
\left.\left(\rho_{\mathrm{cp}}-\rho_{i}\right)\right],
\end{gathered}
$$

$A=\frac{d}{m} \cdot \frac{1}{c_{k} \cdot Q_{n} \cdot \mu}$ - coefficient of connection of parameters characterizing physical and mechanical properties of grain;

$f=\frac{P_{\mathrm{H}}^{3.5} \cdot \tau^{2}}{\mathrm{TH} \cdot \rho^{0.5}}$ - Independent parameter communication function $(\mathrm{P}, \mathrm{T}, \tau, \rho)$;

$\frac{\partial f}{\partial P} ; \frac{\partial f}{\partial \mathrm{T}} ; \frac{\partial f}{\partial \tau} ; \frac{\partial f}{\partial \rho}$ - private derivative functions of communication according to independent parameters;

Pcp, Tcp , $\tau$ cp , $\rho \mathrm{cp}$ - average values of parameters in considered intervals.

Values of the partial derivatives of the communication function $f=\frac{P_{H}^{3,5} \tau^{2}}{T_{H} \rho^{0,5}}$ independent parameters are calculated at substitution of nominal values of independent parameters in them: $\mathrm{P}=20 \cdot 103 \mathrm{~Pa} ; \mathrm{T}=328^{\circ} \mathrm{K} ; \tau=360 \mathrm{~s} ; \rho=0,26 \mathrm{~kg} / \mathrm{m} 3$ :

$$
\begin{gathered}
\frac{\partial f}{\partial P}=\frac{7 \tau^{2} P_{H}^{5 / 2}}{2 T_{H} \sqrt{\rho}}=16 \cdot 10^{13} ; \quad \frac{\partial f}{\partial T}=\frac{-\tau^{2} \cdot P_{H}^{3,5}}{T_{H} \sqrt{\rho}}=-87,0 \cdot 10^{16} ; \\
\frac{\partial f}{\partial \tau}=\frac{2 \tau P_{H}^{3,5}}{T_{H} \sqrt{\rho}}=0,48 \cdot 10^{16} ; \frac{\partial f}{\partial \rho}=\frac{-\tau^{2} P_{H}^{3,5}}{2 T_{H} \rho^{3 / 2}}=-167 \cdot 10^{16}
\end{gathered}
$$

Experimental data obtained in operation and given in Table 2 are used to find average values of processing parameters.

Table 2. Experimental values of parameters of vacuum-sorption humidification process.

\begin{tabular}{|l|c|c|c|c|c|}
\hline $\begin{array}{c}\text { Designation } \\
\text { parameters }\end{array}$ & Dimension & \multicolumn{4}{|c|}{ Values of parameters } \\
\hline $\mathrm{S}_{j}($ time $-\tau)$ & $\mathrm{s}$ & 120 & 240 & 360 & 480 \\
\hline$X_{j}($ pressure $-P)$ & $\mathrm{Pa}$ & $2510^{3}$ & $3010^{3}$ & $4010^{3}$ & $5010^{3}$ \\
\hline$Z_{j}$ (temperature $\left.-T\right)$ & $K$ & 328 & 330 & 331 & 333 \\
\hline$R_{\mathrm{j}}($ density $-\rho)$ & $\mathrm{kg} / \mathrm{m}^{3}$ & $1610^{-2}$ & $1910^{-2}$ & $2610^{-2}$ & $3610^{-2}$ \\
\hline
\end{tabular}

After substitution of numerical values of parameters corresponding to, for example, processing time equal to 4 minutes into expression (7), value of Yi:

$$
\mathrm{Yi}=22 \cdot 106+0.1 \cdot(12.6 \cdot 10-10 / 50.7) \cdot[16 \cdot 1016(30-20)-87 \cdot 1016(330-328)+
$$




$$
0.48 \cdot 1016(240-360)-167 \cdot 1016(0.19-0.26)]=23.4 \cdot 106, \mathrm{~Pa}
$$

The built graphic dependencies of the grain elasticity modulus - EK on the time of vacuum-sorption moisturization taking into account the stochastic nature of the grain shells (microcapillar porosity of grain $\Pi$ varies from 0.1 to 0.4 ) are shown in Figure 1.

The obtained Yi value (8) is within the limits of its minimum and maximum values (6), but in order to establish similarity of operation of sub-systems and in stochastic sense it is necessary that the sums of relative changes (errors) of these complexes, taking into account their correlation from the dimension of performance characteristics, are within the limits of relative tolerances for changes in these characteristics.

Numerical values of independent parameter errors were:

$$
\begin{gathered}
\delta_{X_{j}}=\frac{X_{j}-X_{j_{0}}}{X_{j}}=(30-20) / 30=0.33, \delta_{Z_{j}}=\frac{Z_{j}-Z_{j}}{Z_{j}}=(330-328) / 330=0.01, \\
\delta_{S_{j}}=\frac{S_{j}-S_{j_{0}}}{S_{j}}=(240-360) / 240=-0.5, \delta_{R_{j}}=\frac{R_{j}-R_{j_{0}}}{R_{j}}=(19-26) / 19=-0.368 .
\end{gathered}
$$

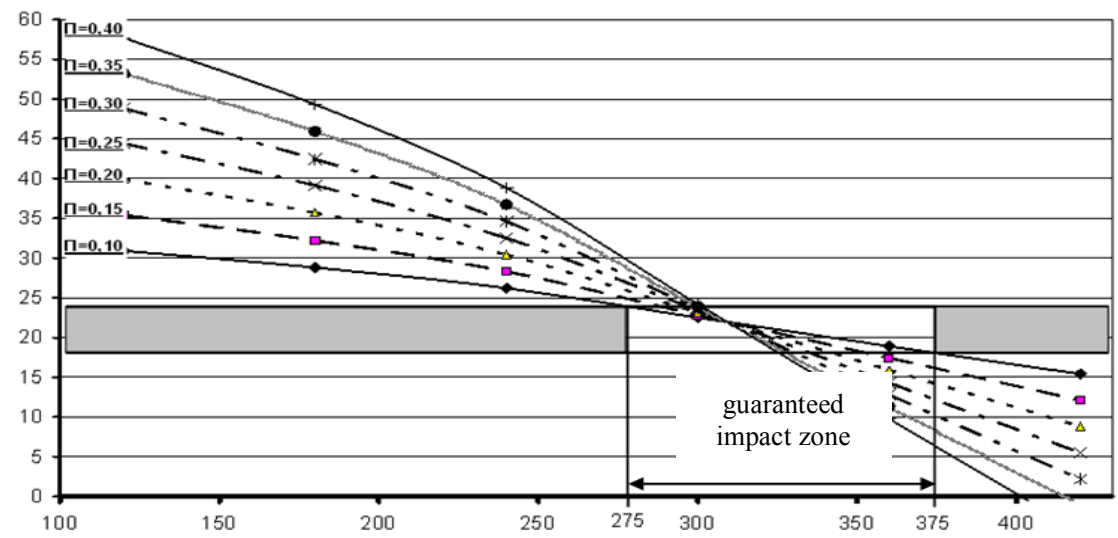

Fig. 1. Changes in the final modulus of elasticity of grain $E_{K}$ from the conditional micro-capillary porosity.

\section{Research and discussion}

Thus, mathematical models of stochastic similarity of functioning of heat-mass transfer processes in porous media (cereal casings) developed on the basis of synergetic approach [1] can be used for control of hygrothermic processes in processing of cereals having stochastic nature of structure [14-17].

\section{References}

1. A. Syrko, Russian journal of general chemistry Oleiades publishing 6.85 p. (2015)

2. V. Pershin, Methodology of similarity of functioning of technical systems: monograph (UTC "Nabla" JÜRGTU (NPI) Mines: YURGUES, Novocherkassk, 2014)

3. L. Larina, D. Ruslyakov, O. Tihonova, Developmen of the control algorithm of processes of intensive hygrothermal imp ct on capillary and porous materials in the conditions of the vacuum, MATEC Web of Conferences 132 (2017) 
4. L. Larina, D. Ruslyakov, O. Tihonova, Investigation of the influence of the microcapillary structure of natural skins on relative humidity in vacuum-sorption humidification, MATEC Web of Conferences 132 (2017)

5. G. Mazza, Anthocyanins in Fruits, Vegetables, and Grains (2018) doi:10.1201/9781351069700

6. F. Shahidi, Ambigaipalan. Journal of Functional Foods 18, 820-897 (2015) doi:10.1016/j.jff.2015.06.018

7. L. Larina, Engineering Bulletin of the Don 3 (2012)

8. N. Gangopadhyay, Food Processing Technologies 20, 10884-10909 (2015)

9. S. Buzoverov, Young scientist 11(22).2, 193-195 (2015)

10. T. Nikiforova, Bread products 5, 50-51 (2014)

11. W. Grajek, Functional Food Product Development (2010) doi:10.1002/9781444323351.ch9

12. Z. Zamaleev, Fundamentals of hydraulics and heat engineering (Lan, SPb, 2014)

13. R. Amarowicz et al., Molecular Nutrition and Food Research 53, 151-183 (2009) doi: $10.1002 / \mathrm{mnfr} .200700486$

14. D.J. Charles, Springer 4, 1-610 (2013).doi:10.1007/978-1-4614-4310-0

15. H. Gazor, International Journal of Food Engineering, 158-162 (2011) https://www.scopus/com 